\title{
Fenologia reprodutiva do araticum e suas implicações no potencial produtivo
}

\author{
Aniela Pilar Campos de Melo*, Alexsander Seleguini, Anderson Fernandes Leite, \\ Eli Regina Barboza de Souza, Ronaldo Veloso Naves
}

Universidade Federal de Goiás, Goiânia, GO, Brasil

*Autor correspondente, e-mail: aniela.pcdmelo@gmail.com

\begin{abstract}
Resumo
O araticum (Annona crassiflora Mart.) é uma frutífera nativa do Bioma Cerrado. Trata-se de espécie frutícola de grande potencial econômico e social. Estudos referentes a fenologia de plantas nativas do bioma Cerrado são escassos e podem ser promissores para um aproveitamento racional e sustentável de suas potencialidades. Assim, o objetivo deste trabalho consistiu na caracterização da fenologia reprodutiva do araticum no Cerrado Goiano. A avaliação das fenofases ocorreu de agosto de 2008 a agosto de 2010, em Goiânia, por meio da contagem de botões florais, flores em antese e frutos formados. A capacidade produtiva foi estimada por meio da taxa de frutificação efetiva. $O$ araticum apresentou um padrão de bianualidade quanto a emissão de flores. A taxa de frutificação efetiva correspondeu a 3,29\%. As fenofases reprodutivas do araticum concentram-se na estação chuvosa. A taxa de frutificação efetiva é muito baixa e dependente de fatores bióticos e abióticos.
\end{abstract}

Palavras-chave: Annona crassiflora Mart., frutífera, floração, frutificação

\section{Araticum reproductive phenology and its implications for productive potential}

\begin{abstract}
The araticum (Annona crassiflora Mart.) is a native fruit from the Cerrado biome, with a great economic and social potential. Studies on Cerrado native plants phenology are scarce and can be promising for a better rational and sustainable exploitation of its potentialities. Thus, the objective of this study was to characterize araticum reproductive phenology in the Cerrado. The phenophases evaluations were carried out from August 2008 to August 2010, in Goiania, Goias, Brazil, through counting of flower buds, flowers in anthesis and fruits formed. The production capacity was estimated through the effective fruiting rate. For flower emission, a biannual pattern was observed for araticum.. The effective fruiting rate observed was $3.29 \%$ and the araticum's reproductive phenophases were concentrated during the rainy season. The effective fruiting rate is considered very low and depends on biotic and abiotic factors.
\end{abstract}

Keywords: Annona crassiflora Mart., flowering, fruit tree, fruiting 
O araticum (Annona crassiflora Mart.) é a anonácea de maior destaque e importância no bioma Cerrado. Os frutos in natura são apreciados pelas comunidades locais e aproveitados por meio da produção de sucos, sorvetes e geléias (Luzia \& Jorge, 2012). Além da riqueza nutricional presente na polpa do fruto (Cardoso et al., 2013; Siqueira et al., 2013), a fração lipidica nas sementes pode ser usada na indústria farmacêutica e de alimentos e para fabricação de biodiesel (Egydio \& Dos Santos, 2011; Luzia \& Jorge, 2012; Sirena et al., 2014).

Estudos referentes a fenologia de plantas nativas do Cerrado são escassos e podem ser promissores para um aproveitamento racional e sustentável de suas potencialidades (Almeida et al., 2014). A fenologia refere-se a definição de fitofases em escala temporal (Biondi et al., 2007). Informações a respeito de brotação, florescimento, frutificação e dispersão de sementes definem os padrões vegetativos e reprodutivos das espécies.

O conhecimento referente as fenofases possui ínumeras vertentes relacionadas à aspectos biológicos, ecológicos e agronômicos. Destaca-se a utilização desses parâmetros para: o planejamento de cruzamentos dirigidos e auxílio na determinação de práticas culturais (Danner et al., 2010; Kuaraksa et al., 2011; Singhal et al., 2011), a definição de estratégias para a recuperação, conservação e manejo de áreas nativas e/ou degradadas (Biondi et al., 2007; Kuaraksa et al., 2011), a compreensão das relações ecológicas entre diferentes espécies (Baver et al., 2012; Françoso et al., 2014) e o estabelecimento da influência dos fatores bióticos e abióticos nos padrões fenológicos das espécies (Reys et al., 2005; Torres \& Galetto, 2011).

O objetivo deste trabalho consistiu na caracterização da fenologia reprodutiva do araticum no Cerrado Goiano.

O estudo foi conduzido na coleção ex-situ de A. crassiflora (16 $6^{\circ} 36^{\prime} \mathrm{S}, 4^{\circ} 16^{\prime} \mathrm{O}$, altitude 727 m.), no município de Goiânia. Esta coleção foi implantada em 2002, simulando um monocultivo, no espaçamento de $5 \times 3$. As mudas foram obtidas por meio de propagação sexuada, sendo as sementes provenientes de matrizes localizadas no estado de Goiás.

O solo na área experimental é classificado como Latossolo Vermelho Escuro. O clima na região é do tipo Aw, apresentando verão chuvoso e inverno seco, com precipitação anual de $1800 \mathrm{~mm}$ e temperatura média de $23^{\circ} \mathrm{C}$. A estação seca predomina nos meses de maio a outubro.

Os dados climatológicos referentes à temperatura, precipitação e umidade relativa nos períodos avaliados foram obtidos na estação evaporimétrica pertencente a Universidade Federal de Goiás, localizada a $1,5 \mathrm{~km}$, da coleção ex-situ.

A caracterização das fenofases reprodutivas foi realizada de agosto de 2008 a agosto de 2010. A avaliação fenológica semanal ocorreu nas 64 plantas presentes na coleção. Durante os ciclos produtivos referentes a 2008/2009 e 2009/2010, as fenofases foram determinadas em função da emissão de botões florais, flores em antese e frutos formados. A capacidade produtiva foi estimada por meio da taxa de frutificação efetiva.

A determinação da quantidade de flores que atingiram a antese foi feita indiretamente por meio de contagem de pétalas no chão, especificamente na projeção da copa. Após a antese, há a abscisão das pétalas, sendo que a cada três pétalas caídas registrou-se uma flor que atingiv a antese (Nascimento et al., 2002).

Os dados referentes a fenologia reprodutiva e atributos climáticos foram avaliados por meio de estatística descritiva (média) e análise de componentes principais (PCA - matriz de correlação).

Independemente do ciclo de avaliação (2008/2009 e 2009/2010), a emissão de botões florais ocorreu em setembro (Figura 1 e 2). Das 64 plantas avaliadas, apenas três não emitiram botões florais em nenhum dos dois ciclos. As plantas que apresentaram um florescimento reduzido no ciclo 2008/2009 obtiveram um florescimento abundante no ciclo seguinte (Figura 1). Cavalcante et al. (2009) relatam que tal comportamento pode ser relativo à padrões de bianualidade desta espécie em relação a produção de flores. Considerando os dois ciclos 
produtivos, verificou-se que, a quantidade de

botões florais por planta variou de 0 a 77 .

O período de floração do araticum,

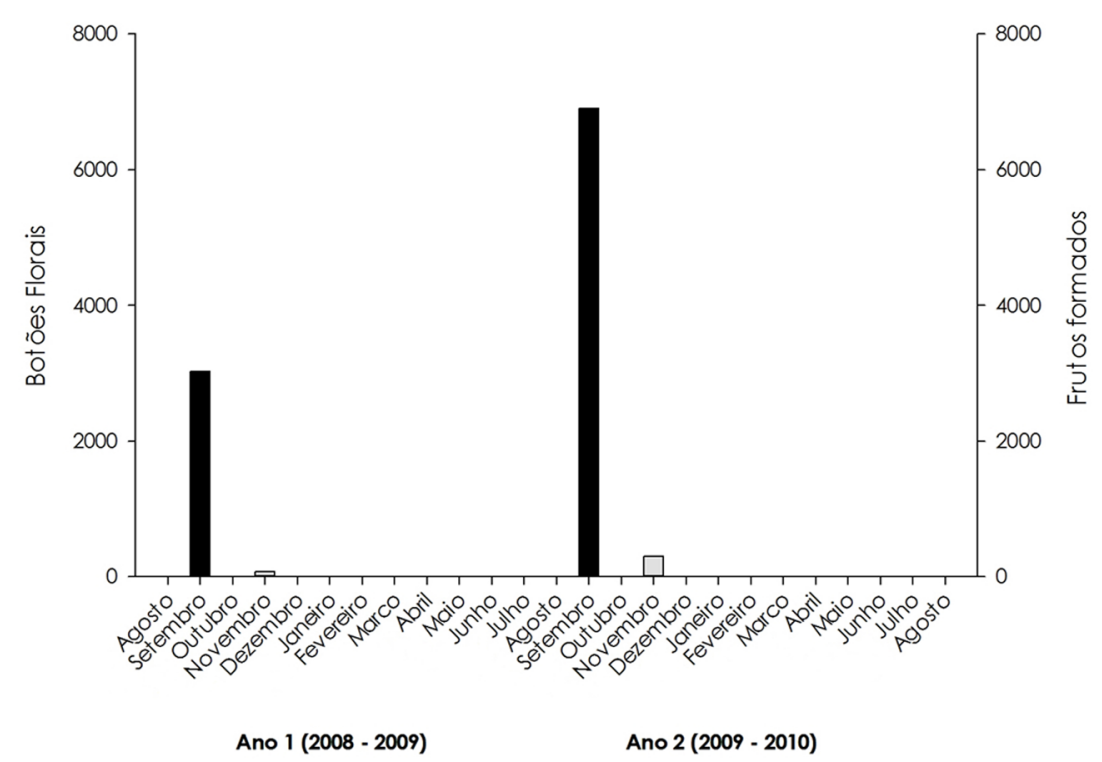

Figura 1. Emissão de botões florais e frutos de plantas de araticum no Cerrado goiano.
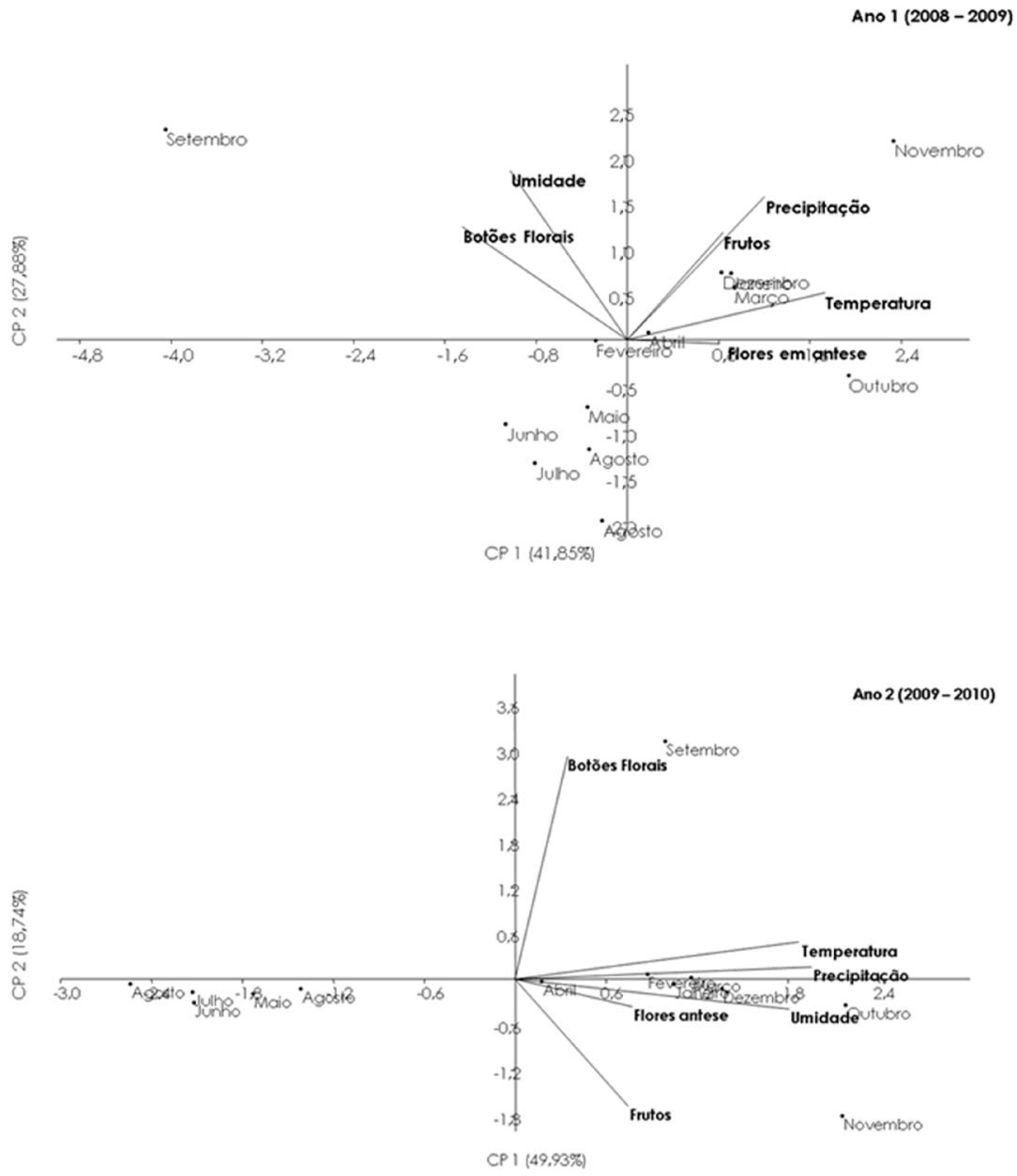

Figura 2. Biplot de variáveis da fenologia reprodutiva (Botões Florais, Flores em antese e Frutos) de plantas de araticum e fatores climáticos (Temperatura, Umidade e Precipitação) em relação aos componentes principais 1 e 2 . 
nos dois ciclos avaliados, coincidiu com o final da estação seca, caracterizado pelo início das chuvas e aumento da umidade relativa (Figura 2 e 3). Tal aspecto também ocorre em outras espécies frutíferas arbustivas-arbóreas do Cerrado, como cagaita (Eugenia dysenterica DC.) (Souza et al., 2008), pequi (Caryocar brasiliense Camb.), mangaba (Hancornia speciosa Goméz) (Batalha \& Mantovani, 2000), pimenta-de-macaco (Xylopia aromatica (Lam.) Mart.) (Camargo et al., 2011) e em florestas semidecíduas (Reys et al., 2005). A combinação entre a redução do estresse hídrico, associado às primeiras chuvas, ao aumento de temperatura e fotoperíodo culmina na indução da floração em florestas tropicais (Opler et al., 1976).

Nos períodos avaliados, a antese iniciou-

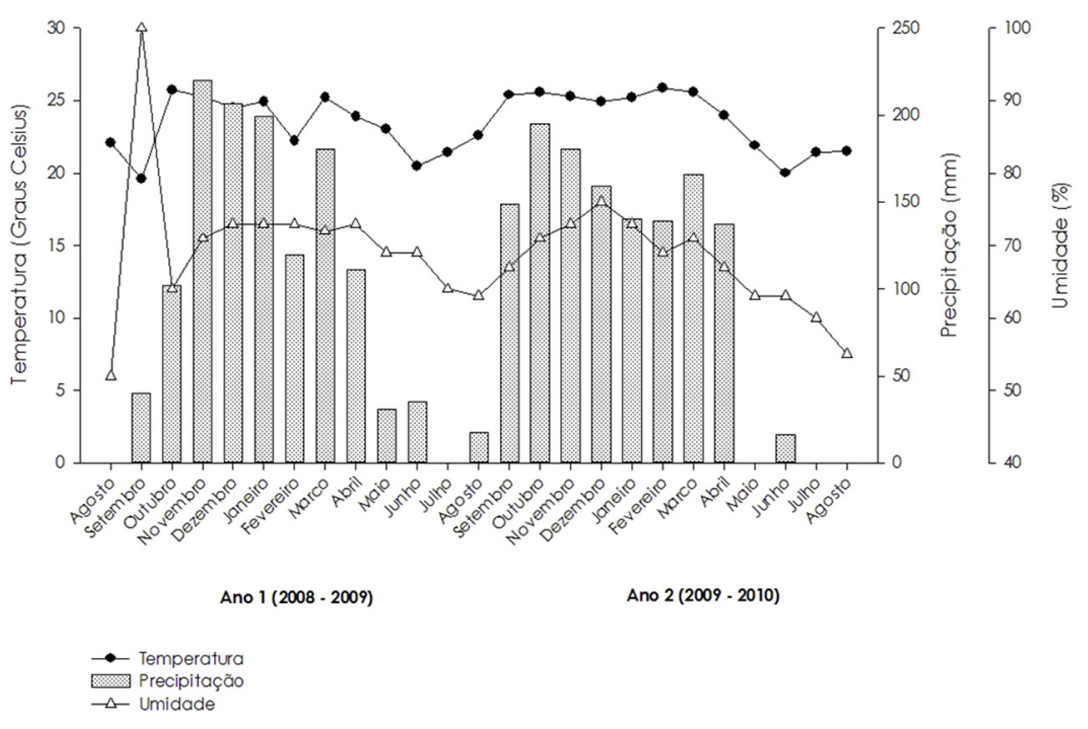

Figura 3. Distribuição de temperatura (Graus Celsius), precipitação (mm) e umidade (\%) no período de floração e frutificação do araticum no Cerrado goiano.

se na primeira quinzena de novembro e o término ocorreu na segunda quinzena de dezembro. $74,84 \%$ dos botões emitidos atingiram à antese (Figura 4). A antese é considerada progressiva e predominantemente matinal (Cavalcante et al., 2009). A. crassiflora é considerada autocompatível, predominantemente alógama e dependente de agentes polinizadores, como os besouros Cyclocephala atricapilla Mannerheim, Cyclocephala laterica Hohne, Cyclocephala octopunctata Burmeister e Trichoton garbei (Geb.) (Braga Filho et al., 2005; Cavalcante et al., 2009).

A emissão de frutos ocorreu predominantemente na segunda quinzena de novembro (Figura 1 e 2). O pico de formação na estação chuvosa pode ser relacionado a estratégia reprodutiva da planta aliada ao oferecimento de alimento para animais dispersores de sementes (Reys et al., 2005). A anta (Tapirus terrestris) e os besouros Oxysternon palaemon (Castelnau 1840), Phanaeus palaeno
(Blanchard 1843), Diabrotics mirabilis (Harold 1877) são considerados dispersores ativos de sementes de araticum. Além de dispersá-las a longas distâncias podem protegê-las contra insetos predadores (Golin et al., 2011).

A taxa de frutificação efetiva nos dois ciclos produtivos correspondeu a 3,29\% (Figura 4). A baixa frutificação natural do araticum é atribuída a diversos fatores relacionados a morfologia e genética floral, a idade da planta, ao suprimento hídrico no solo, ao baixo número de polinizadores, a ocorrência de insetos-praga em flores e frutos e a presença de ventos intensos que provocam a queda de frutos em desenvolvimento (Braga Filho et al., 2005; Cavalcante et al., 2009). Individuos adultos de Telemus chapadanus (Casey 1922) perfuram botões florais e flores totalmente abertas, provocando a quedas destas estruturas e favorecendo a entrada de patógenos (Braga Filho et al., 2005). Cerconota anonella (Sepp.) e Spermologus funereus provocam danos nos 
frutos tornando-os impróprios ao consumo (Braga Filho et al., 2007; Leite et al., 2012). Os besouros S. rufus Boheman, 1943 e Lydamis variegatus (Casey 1922), a vespa Bephratelloides pomorum (Fab.) são predadores de sementes (Braga Filho et al., 2007; Golin et al., 2011).

Foram produzidos por planta cerca de 2,8 frutos. No entanto, entre todas as plantas

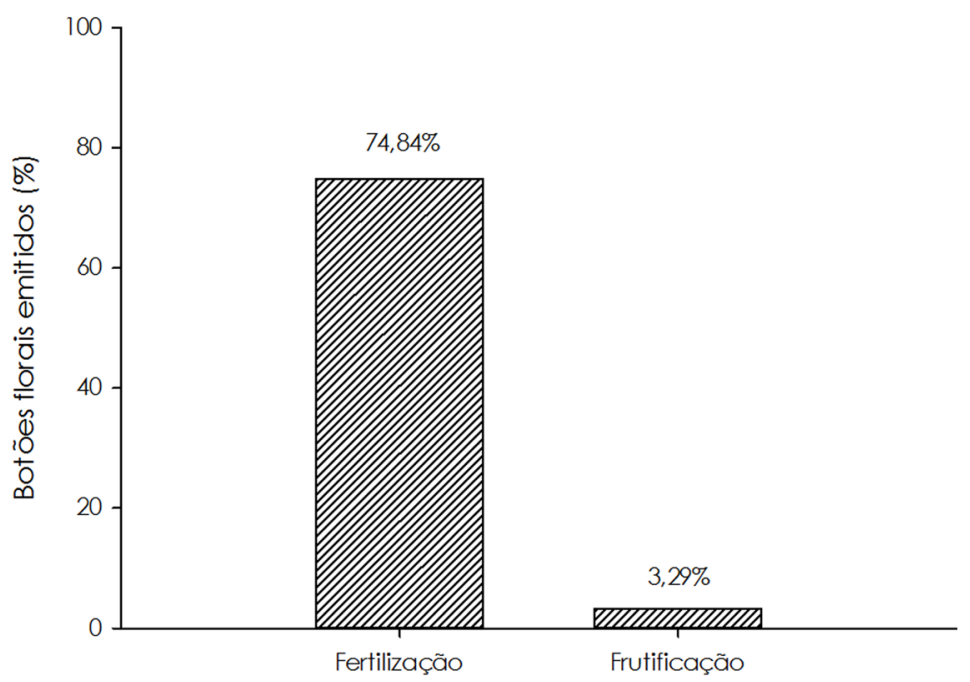

Figura 4 - Relação entre botões florais, flores fertilizadas e frutificação efetiva em plantas de araticum no Cerrado goiano

avaliadas, duas se destacaram por produzirem sozinhas, nos dois ciclos, quase $30 \%$ dos frutos formados. Segundo Braga Filho et al. (2009) esse comportamento é comum em araticunzeiros. Essa heterogeneidade no ciclo reprodutivo é um reflexo da variabilidade genética decorrente da propagação sexuada utilizada no estabelecimento da coleção (Danner et al., 2010). Desta forma, tais plantas apresentam potencial para serem utilizadas para o cruzamento em programas de melhoramento genético e para o fornecimento de estruturas para propagação vegetativa.

Pode-se concluir que, as fenofases reprodutivas do araticum concentram-se na estação chuvosa. A taxa de frutificação efetiva é muito baixa e dependente de fatores bióticos e abióticos.

\section{Referências}

Almeida, J., Santos, J.A., Alberton, B., Torres, R.S., Morellato, L.P.C. 2014. Appying machine learning based on multiscale classifiers to detect remote phenology patterns in Cerrado savanna trees. Ecological Informatics 23:49-61.

Batalha, M.A., Mantovani, W. 2000. Reproductive phenological patterns of cerrado plant species at the Pe-de-Gigante reserve (Santa Rita do Passa Quatro, SP, Brazil): A comparison between the herbaceous and woody floras. Revista Brasileira de Biologia 60:129-145.

Baver, D., Goetz, M. N. B., Muller, A., Schmitt, J. L . 2012. Fenologia de três espécies de Myrsine L. em floresta secundária semidecídua no Sul do Brasil. Revista Árvore 36:859-868.

Biondi, D., Leal, L., Batista, A. 2007. Fenologia do florescimento e frutificação de espécies nativas dos Campos. Acta Scientiarum. Biological Sciences 29:269-276.

Braga Filho, J. R., Veloso, V. R. S., Naves, R. V., Nascimento, J. L. 2005. Danos de Telemus chapadanus (Casey 1922) sobre o florescimento do araticum (Annona crassiflora Mart.) no Estado de Goiás. Pesquisa Agropecuária Tropical 35: 2529.

Braga Filho, J. R., Veloso, V. R. S., Naves, R. V., Nascimento, J. L., Chaves, L.J. 2007. Danos causados por insetos em frutos e sementes de araticum (Annona crassiflora Mart., 1841) no cerrado de Goiás. Bioscience Journal 23:21-28.

Braga Filho, J. R., Veloso, V. R. S., Chaves, L.J., Nascimento, J. L., Aguiar, A.V. 2009. Produção de frutos e caracterização de ambientes de ocorrência de plantas nativas de araticum no cerrado de Goiás. Revista Brasileira de Fruticultura 31: 461-473.

Camargo, M.G.G., Souza, R.M., Morellato, L.P.C. 2011 . Effects of environmental conditions associated to the cardinal orientation on the reproductive phenology of the cerrado savanna tree Xylopia aromatica (Annonaceae). Anais da Academia 
Brasileira de Ciências 83:1007-1019.

Cardoso, L.M., Oliveira, D.S., Bedetti, S.F., Martino, H.S.D., Pinheiro-Sant'ana, H.M. 2013. Araticum (Annona crassiflora Mart. ) from the Brazilian Cerrado: chemical composition and bioactive compounds. Fruits 68: 121-134.

Cavalcante, T., Naves, R., Franceschinelli, E., Da Silva, R. 2009. Polinização e formação de frutos em araticum. Bragantia 68:13-21.

Danner, M.A., Citadin, I., Sasso, S.A.Z., Sachet, M. R., Ambrósio, R. 2010. Fenologia da floração e frutificação de mirtáceas nativas da floresta com araucária. Revista Brasileira de Fruticultura 32: 291 295.

Egydio, A.P.M., Dos Santos, D.Y.A.C. 2011. Underutilized Annona Species from the Brazilian Cerrado and Amazon Rainforest: A Study on Fatty Acids Pro- file and Yield of Seed. Economy Botany 65:329-333.

Françoso, R., Guaraldo, A. de C., Prada, M., Paiva, A.O., Mota, E.H., Pinto, J.R.R. 2014. Fenologia e produção de frutos de Caryocar brasiliense Cambess. E Enterolobium gummiferum (Mart.) J.F.Macbr. em diferentes regimes de queima. Revista Árvore 38:579-590

Golin, V., Santos-Filho, M., Pereira, M.J.B. 2011. Dispersão e predação de sementes de araticum no Cerrado de Mato Grosso, Brasil. Ciência Rural 41:101-107.

Kuaraksa, C., Elliott, S., Hossaert-Mckey, M. 2012. The phenology of dioecious Ficus spp. tree species and its importance for forest restoration projects Forest Ecology and Management 265:82-93.

Leite, G.L.D., Souza, M.F., Souza, P.N.S., Fonseca, M.M.; Zanuncio, J. C. 2012. The bagging of Annona crassiflora fruits to control fruit borers. Acta Scientiarum. Agronomy 34:253-257.

Luzia, D.M.M., Jorge, N. 2013. Bioactive substance contents and antioxidant capacity of the lipid fraction of Annona crassiflora Mart. Seeds. Industrial Crops and Products 42:231-235.

Nascimento, T. B., Gazel Filho, A.B., Santos, J.A. 2002. Fenologia da gravioleira (Annona muricata) em área do Cerrado do Amapá, Brasil. Acta Amazonica 32:367-376.

Opler, P.A., Frankie, G.W., Baker, H.G. 1976.Rainfall as a factor in the release, timing, and synchronization of anthesis by tropical trees and shrubs. Journal of Biogeography 3:231-236.

Reys, P., Galleti, M., Morellato, L. 2005. Fenologia reprodutiva e disponibilidade de frutos de espécies arbóreas em mata ciliar no rio Formoso, Mato Grosso do Sul. Biota Neotropica 5:1-10.
Singhal, V.K., Salwan, A., Kumar, P., Kaur, J. 2011. Phenology, Pollination, Breeding system of Aegle marmelos (Linn.) Correa. (Rutaceae) from India. New Forests 42:85-100.

Siqueira, E.M.A., Rosa, F.R., Fustinoni, A.M., Sant'ana, L.P., Arruda, S.F. 2013. Brazilian savanna fruits contain higher bioactive compounds content and higher antioxidant activity relative to the conventional red delicious apple. Plos One 8: 1-7.

Sirena, J.T., Flach, A., Costa, L.A.M.A., Silva, C.S.F., Peixoto, C.R.M., Moura, N.F. 2014. Chemical composition of the essential oil from Annona crassiflora. Chemistry of Natural Compounds 50: 543-544.

Souza, E.R.B., Naves, R.V., Borges, J.D., Vera, R., Fernandes, E.P., Silva, L.B., Trindade, M.G. 2008. Fenologia de cagaiteira (Eugenia dysenterica DC.) no Estado de Goiás. Revista Brasileira de Fruticultura 30:1009-1014.

Torres, C., Galetto, L. 2011. Flowering phenology of co-occurring Asteraceae: a matter of climate, ecological interactions, plant attributes or of evolutionary relationships among species? Organisms Diversity \& Evolution 1 1:9-19. 